\title{
ESTUDO RETROSPECTIVO DE 50 CASOS DE CÓLICA EM EQUINOS ATENDIDOS NO HOSPITAL VETERINÁRIO DA FCAV - UNESP, NO PERÍODO DE SETEMBRO DE 2004 A JULHO DE 2005
}

\author{
Paula Alessandra Di Filippo, ${ }^{1}$ Rodrigo Norberto Pereira, ${ }^{2}$ João Henrique Perotta, ${ }^{3}$ \\ Aracelle Elisane Alves, ${ }^{4}$ Deborah Penteado Martins Dias ${ }^{5}$ e Áureo Evangelista Santana ${ }^{6}$ \\ 1. Professora doutora associada do Centro de Ciências e Tecnologias Agropecuárias da Universidade Estadual Fluminen "Darcy Ribeiro"/UENF, \\ Campos dos Goytacazes, RJ. - E-mail: paula difilippo@yahoo.com.br \\ 2. Professor doutor adjunto do Centro de Ciências Agropecuárias da Universidade Federal da Paraíba/UFPB, Areia, PB \\ 3. Professor mestre da Faculdade Evangélica do Pará, Curitiba, PR \\ 4. Doutora em Cirurgia Veterinária pela Faculdade de Ciências Agrárias e Veterinárias/FCAV/Unesp, Jaboticabal, SP \\ 5. Doutoranda em Cirurgia Veterinária pela Faculdade de Ciências Agrárias e Veterinárias/ FCAV/Unesp, Jaboticabal, SP \\ 6. Professor adjunto do Departamento de Clínica e Cirurgia Veterinária da FCAV/Unesp, Jaboticabal, SP.
}

\section{RESUMO}

Revisaram-se os dados de cinquenta equinos com cólica submetidos à laparotomia exploratória, atendidos no Hospital Veterinário da FCAV/Unesp, campus de Jaboticabal, entre setembro de 2004 e julho de 2005. Animais das raças Brasileira de Hipismo (28\%), Paint Horse (16\%) e Quarto de Milha (12\%) foram os mais acometidos. Fêmeas mostraram-se mais propensas à cólica (46\%) que machos não castrados (28\%) e castrados (26\%). Animais com média de idade entre 2 e 10 anos foram prevalentes (64\%). Em geral, as três causas mais comuns de cólica foram deslocamento (22\%), compactação do cólon maior $(16 \%)$ e hérnia inguinoescrotal $(12 \%)$. Dos cinquenta animais com cólica submetidos à

PALAVRAS-CHAVES: Cavalo, cólica, laparotomia, prognóstico. laparotomia, 27 (54\%) sobreviveram e $23(46 \%)$ foram a óbito ou sacrificados. Dentre os 27 sobreviventes, 21 (78\%) apresentavam lesões no intestino grosso (IG) e 6 (22\%) no intestino delgado (ID). Entretanto, dos 23 animais que foram a óbito ou sacrificados, 15 $(65,21 \%)$ apresentavam lesões no ID e $8(34,78 \%)$ no IG. O tempo médio de evolução do distúrbio até que os animais recebessem atendimento especializado foi de 20,13 horas para os animais com lesões no IG e de 13,29 horas para os equinos com lesões no ID. A demora no atendimento reduz as chances de recuperação dos animais e contribui para o expressivo número de procedimentos cirúrgicos realizados.

\section{ABSTRACT}

\section{RETROSPECTIVE STUDY OF 50 EQUINE COLIC CASES PRESENTED TO THE VETERINARY HOSPITAL OF FCAV-UNESP BETWEEN 2004 AND 2005}

The medical records of 50 equine gastrointestinal colic cases submitted to laparatomy presented to the Veterinary Hospital of FCAV-UNESP between 2004 and 2005 were reviewed. The breeds with higher predisposition to colic were Brasileira de Hipismo (28\%), Paint Horse (16\%) and Quarter Horse (12\%). Females $(46 \%)$ were more prone to colic than stallions $(28 \%)$ and geldings (26\%). Overall, the 3 most common causes of colic were large colon displacement (22\%), large colon impaction
(16\%), and inguinal hernia. Animals with average age between two and ten years were the most affected (64\%). From the 50 animals with colic submitted to laparatomy, 27 (54\%) survived and $23(46 \%)$ were sacrificed or died. Among the 27 survivors, $21(78 \%)$ had lesions on the large intestine (LI) and six (22\%) on the small intestine (SI). However, from the 23 animals that died or were euthanized, 15 (65.21\%) showed damage on SI and eight $(34.78 \%)$ on LI. The mean time between the first symptoms and 
the carrying out of specialized treatment was 20.13 hours to the animals with damage on LI and 13.29 hours to the equines with damage on SI. In conclusion, the delay on the admission reduces

KEYWORDS: Colic, horse, laparotomy, prognosis.

\section{INTRODUÇÃO}

As doenças que envolvem o sistema digestório, tais como as cólicas, as diarréias e as enterotoxemias, representam $50 \%$ dos problemas médicos que resultam na morte de cavalos adultos (GONÇALVES et al., 2002). A cólica, por envolver fatores - de natureza e grandeza - distintos, apresenta patogenia que pode variar desde um distúrbio passageiro a um episódio complexo e de difícil resolução, constituindo-se na doença mais comum e severa (ALVES, 1994).

Apesar dos avanços em relação aos métodos de diagnóstico, às técnicas anestésicas e cirúrgicas e ao acompanhamento intensivo no pós-operatório, a mortalidade permanece alta. É exigida do médico veterinário ação muito eficaz para impulsionar o correto tratamento, em curto espaço de tempo (THOEFNER et al., 2003). Além do mais, embora as manifestações clínicas dos equinos com abdômen agudo guardem certa semelhança, a etiologia, a patofisiologia e o prognóstico podem ser extremamente diferentes. Por isso, é necessário que o clínico seja capaz de diferenciar casos simples, que podem ser tratados de modo conservativo, daqueles cujos animais apresentam lesões gastrintestinais graves e que podem evoluir para um colapso circulatório e, fatalmente, para a morte.

Estima-se que a incidência anual de cólica nos Estados Unidos seja de 4,2 casos/100 cavalos a um custo aproximado de 115,3 milhões de dólares (MEHDI \& MOHAMMAD, 2006). Na Grã-Bretanha foi observada incidência de 7,2 casos/100 animais por ano e no Irã 8,6\% (HILLYER et al., 2001). Os casos que requerem tratamento cirúrgico resultam em um maior número de óbitos do que os casos tratados clinicamente. A letalidade atinge $13 \%$ dos casos não submetidos à cirurgia e $31 \%$ nos casos que requerem intervenção cirúrgica (KANEENE et al., 1997). As porcentagens dos episódios de cólica que resultam em cirurgia variam de 1,4\% a $6,3 \%$, segundo os plantéis analisados e o tipo de the animals' chances of recovery and contributes to the expressive number of surgical procedures performed.

cólica apresentada (TINKER et al., 1997; TRAUBDARGATZ et al., 2001).

Equinos com histórico de cólicas recorrentes apresentam risco 3,6 vezes maior de serem acometidos por novo episódio (TINKER et al., 1997). Estudo realizado por TRAUB-DARGATZ et al. (2001) revelou taxa de reincidência de $11 \%$ e por VAN DEN BOOM \& VAN DER VELDEN (2001) taxa de 16\%. O histórico de episódio anterior de cólica não ajuda a identificar o mecanismo patofisiológico da cólica e também não é um fator de risco que possa ser alterado. Porém, a associação de um episódio de cólica com um episódio subsequente é uma informação importante para aqueles que manejam os equinos (COHEN, 1997).

Peculiaridades anatômicas, tais como a pequena capacidade volumétrica do estômago, quando comparada com outras espécies domésticas, a incapacidade de regurgitar, dada a musculatura muito desenvolvida do cárdia, e a ausência do centro do vômito no sistema nervoso central, bem como o longo mesentério associado ao jejuno, que favorece as torções, além dos segmentos intestinais com diminuição abrupta do diâmetro do lume, como a flexura pélvica e a transição para o cólon menor, que favorecem o acúmulo de alimentos, e ainda de uma mucosa retal frágil, predisposta a rupturas, tudo isso predispõe os equinos a distúrbios gastrentéricos (PEIRÓ \& MENDES, 2004). Entretanto, outros fatores frequentemente associados a alterações no manejo, na atividade física ou na dieta, infestações parasitárias e fatores intrínsecos, tais como sexo, raça e idade, também tornam o equino propenso a episódios de cólica (LACERDA NETO et al., 1989).

Diante dessas observações, o presente estudo teve o objetivo de descrever o perfil de cinquenta equinos com cólica submetidos à laparotomia exploratória, atendidos no Hospital Veterinário da FCAV/ UNESP, Jaboticabal, SP, no período de setembro de 2004 a julho de 2005. 


\section{MATERIAL E MÉTODOS}

Entre setembro de 2004 a julho de 2005, cinquenta equinos foram encaminhados ao Hospital Veterinário da FCAV, Unesp, Jaboticabal para tratamento da síndrome cólica. Confirmou-se o diagnóstico de cólica cirúrgica através de exame físico, exame transretal, abdominocentese, presença de refluxo por sonda nasogástrica, exploração cirúrgica, ou quando estes não podiam mais ser controlados, medicamente.

Após preparação preoperatória rotineira, todos os animais receberam xilazina ${ }^{1}$ (média de $0,5 \mathrm{mg} \mathrm{kg}^{-1}$ ) via intravenosa (IV) como medicação pré-anestésica. Ato contínuo, procedeu-se à infusão sob pressão de éter gliceril guaicol ${ }^{2}$ a $10 \%(100 \mathrm{mg}$ $\left.\mathrm{kg}^{-1}, \mathrm{IV}\right)$ e midazolam ${ }^{3}\left(0,05 \mathrm{mg} \mathrm{kg}^{-1}, \mathrm{IV}\right)$, seguidos de cetamina ${ }^{4}\left(2 \mathrm{mg} \mathrm{kg}^{-1}, \mathrm{IV}\right)$. Após a intubação orotraqueal, a manutenção foi feita com halotano volatizado em $15 \mathrm{ml} / \mathrm{kg}$ de oxigênio, em circuito anestésico semifechado com ventilação espontânea, sendo os animais posicionados em decúbito dorsal. Durante o procedimento cirúrgico, administraramse 5 a $10 \mathrm{ml} / \mathrm{kg} / \mathrm{min}$, IV, de solução de ringer com lactato para auxiliar a manutenção da pressão arterial média, entre 70 e $100 \mathrm{mmHg}$.

No pós-operatório, instituiu-se terapia antimicrobiana com penicilina benzatina ${ }^{5}$, na dose de $30.000 \mathrm{UI} \mathrm{kg}^{-1}$, via intramuscular (IM), a cada 48 horas, num total de três aplicações. Como analgésico e anti-inflamatório, administrou-se flunixin meglumine $^{6}$ na dose de $0,5 \mathrm{mg} \mathrm{kg}^{-1}$, IV, a cada 12 horas, durante três dias. Reposições hidroeletrolíticas foram realizadas com solução de ringer com lactato e solução fisiológica, calculadas em função das taxas de manutenção e reposição hídrica dos animais. Os fármacos selecionados e a duração de administração variaram em função da gravidade da enfermidade e resposta ao tratamento. $\mathrm{O}$ ensaio recebeu aprovação da Comissão de Ética e Bem-Estar Animal (protocolo $\mathrm{n}^{\mathrm{o}}$ 013332-07).

1 Sedazine - Fort Dodge.

2 Éter gliceril guaiacol - Henrifarma.

3 Midazolam - Hipolabor.

4 Dopalen - Vetbrands.

5 Pentabiótico Reforçado - Fort Dodge.

6 Flunixina Injetável - UCB S.A.

\section{RESULTADOS E DISCUSSÃO}

Na Tabela 1 estão expressos os dados relativos à idade, ao sexo e à raça dos cinquenta equinos com cólica avaliados e respectivas porcentagens.

Houve predomínio de animais com cólica da raça Brasileira de Hipismo (BH), o que foi associado à localização geográfica do centro de referência, margeado por fazendas de criação de animais da referida raça. Ensaio realizado por WHITE \& LESSARD (1986) revelou predomínio de animais da raça Árabe e, segundo COHEN et al. (1995), tal achado deveu-se às características de manejo dos animais. Entretanto, em ensaio desenvolvido por TINKER et al. (1997), animais da raça Puro Sangue Inglês foram os mais acometidos.

Dos cinquenta animais avaliados, $46 \%$ eram fêmeas, $28 \%$ eram machos não castrados e $26 \%$ machos castrados. Sabe-se que os haras do interior do país abrigam principalmente fêmeas reprodutoras. Os machos, castrados ou não, são encaminhados aos grandes centros, onde participam de provas desportivas, o que explica os resultados obtidos neste ensaio. Os resultados diferem dos de ABUTARBUSH et al. (2005), os quais observaram maior número de machos não castrados. Apesar da existência de inúmeros tipos de cólica e da influência dos distúrbios sexo dependentes, segundo MEHDI \& MOHAMMAD (2006), há muitas controvérsias entre os resultados obtidos por diferentes pesquisas. Dos 3.848 equinos com cólica avaliados por KANEENE et al. (1997), os machos não castrados foram os mais acometidos. Entretanto, no mesmo ano, COHEN \& PELOSO observaram, em uma população de 1.214 animais com cólica, predomínio de machos castrados.

Na Tabela 1, observa-se que os animais com idade entre dois e dez anos (64\%) foram os mais acometidos. Resultados semelhantes foram obtidos por COHEN et al. (1999). Segundo TINKER et al. (1997), certos tipos de cólica são mais frequentes em determinadas categorias de idade. As resultantes da obstrução do intestino por enterólitos acometem principalmente animais com idade superior a onze anos. Compactação e deslocamento de cólon maior são frequentemente observados em animais com idade entre sete e oito anos. Entretanto, as chances da ocorrência de torções e estrangulamentos do intestino delgado e 
cólon maior diminuem com a idade. As cólicas que requerem tratamento cirúrgico são mais frequentes em animais idosos. Ainda segundo esses autores, a relação entre idade e o surgimento do episódio de cólica é complexa. A idade pode ser um indicador da utilização do animal, do nível de atividade e do tipo e quantidade de alimento, que constantemente são incriminados no desencadeamento do distúrbio.
Corroborando as afirmações anteriores, observa-se, na Tabela 2, que os principais achados clínico-cirúrgicos foram compactação e deslocamento de cólon maior, sendo a média de idade dos animais acometidos de 7,5 e 8,6 anos, respectivamente.

Os dados relativos às causas da cólica, à duração dos sinais antes da apresentação e evolução do processo após a laparotomia exploratória estão expressos na Tabela 2.

TABELA 1. Raça, sexo e idade dos cinquenta equinos com cólica atendidos no Hospital Veterinário da FCAV, Unesp, campus de Jaboticabal no período de setembro de 2004 a julho de 2005

\begin{tabular}{|c|c|c|c|c|c|c|c|c|}
\hline \multicolumn{3}{|l|}{ Raça } & \multicolumn{3}{|c|}{ Sexo } & \multicolumn{3}{|c|}{ Idade } \\
\hline & $n$ & $\%$ & & $n$ & $\%$ & & $n$ & $\%$ \\
\hline Brasileiro de Hipismo & 14 & 28 & & & & & & \\
\hline Paint-Horse & 8 & 16 & & & & & & \\
\hline Quarto de Milha & 6 & 12 & & & & & & \\
\hline Anglo Árabe & 5 & 10 & & & & & & \\
\hline Mangalarga Marchador & 4 & 8 & Fêmeas & 23 & 46 & $<2$ anos & 8 & 16 \\
\hline Puro Sangue Inglês & 4 & 8 & Machos castrados & 13 & 26 & 2-10 anos & 32 & 64 \\
\hline West-Fallen & 3 & 6 & Machos não castrados & 14 & 28 & $>10$ anos & 10 & 20 \\
\hline Sem raça definida & 3 & 6 & & & & & & \\
\hline Puro Sangue Lusitano & 1 & 2 & & & & & & \\
\hline Sela Belga & 1 & 2 & & & & & & \\
\hline Apaloosa & 1 & 2 & & & & & & \\
\hline
\end{tabular}

$n=$ numero de animais.

TABELA 2. Causas, duração dos sinais da síndrome antes do atendimento e evolução do processo de cinquenta equinos com cólica atendidos no Hospital Veterinário da FCAV, Unesp, campus de Jaboticabal no período de setembro de 2004 a julho de 2005

\begin{tabular}{|c|c|c|c|c|c|c|c|}
\hline \multirow{3}{*}{ Diagnóstico } & \multirow[b]{3}{*}{$n$} & & \multirow{3}{*}{$\begin{array}{c}\text { Duração dos sinais } \\
\text { antes da apresenta- } \\
\text { ção (horas) }\end{array}$} & \multicolumn{4}{|c|}{ Evolução do processo } \\
\hline & & & & \multicolumn{2}{|c|}{ Sobreviveram } & \multicolumn{2}{|c|}{ Sacrificados/Óbitos } \\
\hline & & $\%$ & & $n$ & $\%$ & $N$ & $\%$ \\
\hline $\begin{array}{l}\text { Encarceramento nefroesplênico de } \\
\text { cólon maior }\end{array}$ & 4 & 8 & 7,25 & 4 & 100 & . & . \\
\hline Compactação de cólon menor & 2 & 4 & 60 & 2 & 100 & . & . \\
\hline Compactação de cólon maior & 8 & 16 & 20,71 & 6 & 75 & 2 & 25 \\
\hline Compactação de íleo & 5 & 10 & 8 & 4 & 80 & 1 & 20 \\
\hline Vólvulo do cólon maior & 3 & 6 & 6,33 & 1 & 33,33 & 2 & 66,66 \\
\hline Deslocamento de cólon maior & 11 & 22 & 17,54 & 8 & 72,72 & 3 & 27,27 \\
\hline Hérnia inguinoescrotal & 6 & 12 & 10,83 & 1 & 16,66 & 5 & 83,33 \\
\hline Hérnia epiploica & 2 & 4 & 25,5 & 1 & 50 & 1 & 50 \\
\hline Vólvulo do intestino delgado & 6 & 12 & 11,42 & . & . & 6 & 100 \\
\hline Sablose do cólon maior & 1 & 2 & 9 & . & . & 1 & 100 \\
\hline Intussuscepção ileocecal & 1 & 2 & 16 & . & . & 1 & 100 \\
\hline Torção gástrica & 1 & 2 & 8 & . & . & 1 & 100 \\
\hline
\end{tabular}

$n=$ número de animais. 
Compactação e deslocamento do cólon maior também foram as alterações mais frequentes observadas por ABUTARBUSH et al. (2005). Segundo esses autores, os principais fatores de riscos associados às obstruções do cólon maior (deslocamentos e compactações) incluem a aerofagia, as mudanças nos regimes regulares de exercícios, a falta da administração de anti-helmínticos por tempo superior a doze meses, o histórico de viagens realizadas a menos de 24 horas e o aumento no tempo de estabulagem dos animais. Esses fatores eram comuns entre os animais ensaiados. Os resultados, entretanto, diferem dos obtidos por MARKUS et al. (2008), que observaram predomínio de quadros de enterolitíase $(62,5)$. Alimentação de má qualidade e em quantidade inadequada, falta de cuidados com a dentição e com o controle de verminoses foram os fatores incriminados por esses autores no desenvolvimento da afecção.

Dos cinquenta animais com cólica submetidos à laparotomia exploratória, 27 (54\%) sobreviveram e 23 (46\%) foram a óbito ou foram sacrificados. Dentre os 27 sobreviventes, $21(78 \%)$ apresentavam lesões no intestino grosso (IG) e 6 (22\%) no intestino delgado (ID). Entretanto, dos 23 animais que foram a óbito ou sacrificados, $15(65,21 \%)$ apresentavam lesões no ID e $8(34,78 \%)$ no IG. Ademais, observa-se na Tabela 2 que o tempo médio de evolução do distúrbio, antes que os animais chegassem ao centro de referência, foi de 20,13 horas para os animais que apresentavam lesões no IG (58\%) e de 13,29 horas para os animais com alterações no ID (42\%).

\section{CONCLUSÕES}

Dos cinquenta animais com cólica atendidos na FCAV no período de setembro de 2004 a julho de 2005, fêmeas da raça Brasileira de Hipismo, com idade entre dois e dez anos, apresentando compactação e deslocamento de cólon maior, foram prevalentes. A demora no atendimento reduz as chances de recuperação dos animais e contribui para o expressivo número de procedimentos cirúrgicos realizados.

\section{AGRADECIMENTO}

À Fundação de Amparo à Pesquisa do Estado de São Paulo (Fapesp), pelo financiamento integral a esta pesquisa (processos no 03/09844-5 e 04/08662-3).

\section{REFERÊNCIAS}

ABUTARBUSH, S. M.; CARMALT, J. L.; SHOEMAKER, R. W. Causes of gastrointestinal colic in western Canada: 604 cases (1992 to 2002), Canadian Veterinary Journal, v. 46, n. 9, p. 800-805, 2005.

ALVES, G. E. S. Anamnese. In: FÓRUM DE GASTROENTEROLOGIA EQÜINA, 1., 1994, Curitiba. Anais... Curitiba: CBCAV, 1994.

COHEN, N. D.; GIBBS, P. G.; WOODS, A. M. Dietary and other management factors associated with colic in horses, Journal American Veterinary Medical Association, v. 215, p. 53-60, 1999.

COHEN, N. D. Epidemiology of colic. Veterinary Clinics of North America: Equine Practice, v. 13, n. 2, p. 191-201, 1997.

COHENN, D.; PELOSO, J. G. Risk factors for history of previous colic and for chronic, intermittent colic in a population of horses. Journal American Veterinary Medical Association, v. 208, p. 697-703, 1996.

COHENN, D.; MATEJKA, P. L.; HONNASC, M.; HOOPER, R. N. Case-control study of the association between various management factors and development of colic in horses. Journal American Veterinary Medical Association, v. 206, p. 667-673, 1995.

GONÇALVES, S.; JULLIAND, V.; LEBLOND, A. Risck factors associated with colic in horses. Veterinary Research, v. 33, n. 6, p. 641-652, 2002.

HILLYER, M. H.; TAYLOR, F. G. R.; FRENCH, N. P. A crosssectional study of colic in horses on Thoroughbred training premises in the British Isles in 1997. Equine Veterinary Journal, v. 33 , n. 4 , p. $380-385,2001$.

KANEENE, J. B.; MILLER, R.; ROSS, W. A.; GALLAGHER, K.; MARTENIUK, J.; ROOK, J. Risk factors for colic in Michigan (USA) equine population. Preventive Veterinary Medicine, v. 30, p. 23-36, 1997.

LACERDA NETO, J. C. et al. Cólica eqüina: diagnóstico e tratamento. Jaboticabal: FUNEP, 1989. p. 111.

MARKUS, D.; LINS, L. A.;VIEIRA, J.; CASTRO JR. J.; N.; C. E. W. Casos de cólica em eqüinos com necessidade de intervenção cirúrgica, em dois centros cirúrgicos na cidade de Porto Alegre, durante o primeiro semestre de 2006. In: CONGRESSO DE INICIAÇÃO CIENTIFICA, 16., 2006, Pelotas. Anais... Pelotas: CCI, 2006.

MEHDI, S.; MOHAMMAD, V. A farm-based prospective study of equine colic incidence and associated risk factors. Journal of Equine Veterinary Science, v. 26, n. 4, p. 171-174, 2006. 
NAPPERT, G.; JOHNSON, P. J. Determination of the acid-base status in 50 horses admitted with colic between December 1998 and May 1999. Canadian Veterinary Journal, v. 42, n. 9, p. 703-707, 2001.

PEIRÓ, J. R.; MENDES, L. C. Semiologia do sistema digestório eqüino: In: FEITOSA, F.L.F. Semiologia veterinária: a arte do diagnóstico. São Paulo: Roca, 2004. p. 139-175.

THOEFNER, M. B.; THOEFNER, M. B.; ERSBOLL, B. K.; JANSSON, N.; HESSEL HOLT, M. Diagnostic decision rule for support in clinical assessment of the need for surgical intervention in horses with acute abdominal pain. Canadian Journal Veterinary Research, v. 67, n. 1, p. 20-29, 2003.

TINKER, M. K.; WHITE, N. A.; LESSARD, P.; THATCHER, C. D.; PELZER, K. D.; DAVIS, B.; CARMEL, D. K. Prospective study of equine colic risk factors. Equine Veterinary Journal, v. 29, n. 6, p. 454-458, 1997.
TRAUB-DARGATZ, J. L.; KOPRAL, C. A.; SEITZINGER, A. H.; GARBER, L. P.; FORDE, K.; WHITE, N. A. Estimate of the national incidence of and operation-level risk factors for colic among horses in the United States, spring 1998 to spring 1999. Journal of the American Veterinary Medical Association, v. 219 , n. 1, p. 67-71, 2001.

VAN DEN BOOM, R.; VAN DER VELDEN, M. A. Short- and long-term evaluation of surgical treatment of strangulating obstructions of the small intestine in horses: a review of 224 cases. The Veterinary Quarterly, v. 23, n. 3, p. 109-115, 2001.

WHITE, N. A.; LESSARD, P. Risk factors and clinical signs associated with cases of equine colic. In: ANNUAL CONVENTION AMERICAN ASSOCIATION, Equine Practitioner Proceedings, v. 32, p. 637-644, 1986.

Protocolado em: 30 abr. 2009. Aceito em: 4 maio 2010. 